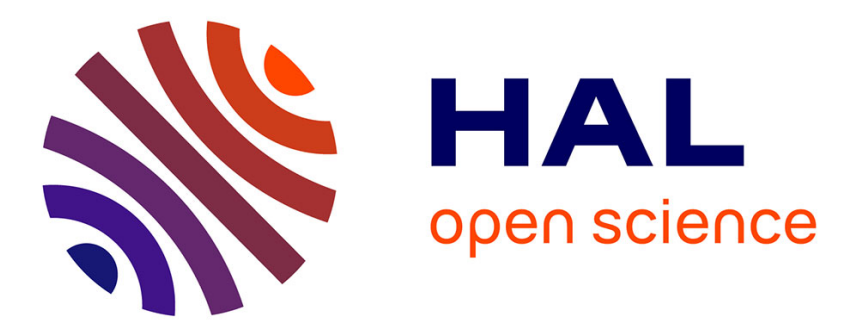

\title{
Shear Rheology Control of Wrinkles and Patterns in Graphene Oxide Films
}

Franco Tardani, Wilfrid Neri, Cécile Zakri, Hamid Kellay, Annie Colin, Philippe Poulin

\section{- To cite this version:}

Franco Tardani, Wilfrid Neri, Cécile Zakri, Hamid Kellay, Annie Colin, et al.. Shear Rheology Control of Wrinkles and Patterns in Graphene Oxide Films. Langmuir, 2018, 34 (9), pp.2996 - 3002. 10.1021/acs.langmuir.7b04281 . hal-01762840

\section{HAL Id: hal-01762840 \\ https://hal.science/hal-01762840}

Submitted on 10 Apr 2018

HAL is a multi-disciplinary open access archive for the deposit and dissemination of scientific research documents, whether they are published or not. The documents may come from teaching and research institutions in France or abroad, or from public or private research centers.
L'archive ouverte pluridisciplinaire HAL, est destinée au dépôt et à la diffusion de documents scientifiques de niveau recherche, publiés ou non, émanant des établissements d'enseignement et de recherche français ou étrangers, des laboratoires publics ou privés.

\section{(1)(1) $\$(0)$}

Distributed under a Creative Commons Attribution - NonCommercial - ShareAlikel 4.0 


\title{
Shear Rheology Control of Wrinkles and Patterns in Graphene Oxide Films
}

\author{
Franco Tardani, ${ }^{*}(1)$ Wilfrid Neri, Cecile Zakri, Hamid Kellay, ${ }^{\dagger}$ Annie Colin, ${ }^{\dagger}$ and Philippe Poulin \\ Centre de Recherche Paul Pascal, CNRS, 33600 Pessac, France
}

\begin{abstract}
Drying graphene oxide (GO) films are subject to extensive wrinkling, which largely affects their final properties. Wrinkles were shown to be suitable in biotechnological applications; however, they negatively affect the electronic properties of the films. Here, we report on wrinkle tuning and patterning of GO films under stress controlled conditions during drying. GO flakes assemble at an air-solvent interface; the assembly forms a skin at the surface and may bend due to volume shrinkage while drying. We applied a

modification of evaporative lithography to spatially define the evaporative stress field. Wrinkle alignment is achieved over $\mathrm{cm}^{2}$ areas. The wavelength (i.e., wrinkle spacing) is controlled in the $\mu \mathrm{m}$ range by the film thickness and GO concentration. Furthermore, we propose the use of nanoparticles to control capillary forces to suppress wrinkling. An example of a controlled pattern is given to elucidate the potential of the technique. The results are discussed in terms of classical elasticity theory. Wrinkling is the result of bending of the wet solid skin layer assembled on a highly elastic GO dispersion. Wavelength selection is the result of energy minimization between the bending of the skin and the elastic deformation of the GO supporting dispersion. The results strongly suggest the possibility to tune wrinkles and patterns by simple physicochemical routes.
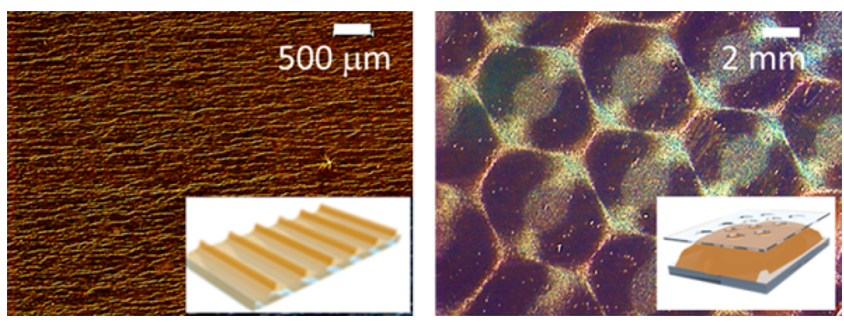

\section{INTRODUCTION}

Graphene oxide (GO) is broadly used for the manufacture of graphene based membranes and films. These are currently investigated for a vast number of technological (electronics, ${ }^{1-3}$ optics, optoelectronics, ${ }^{1,4}$ filtration systems ${ }^{5}$ ) and biotechno logical (biomedical devices and tissue engineering ${ }^{6}$ ) applica tions.

The oxygen containing groups make GO soluble in water and thus easy to handle under environmentally friendly conditions. Graphene like properties are quickly restored after chemical $^{7}$ or thermal ${ }^{8}$ reduction. Moreover, the large shape anisotropy of the sheets assures liquid crystalline (LC) behavior even under dilute conditions. ${ }^{9-11}$ Under shear, GO sheets tend to align and flatten due to the suppression of thermal undulations. ${ }^{12}$ Exotic assemblies can be expected if instabilities set up during deposition, by analogy to lamellar phases. ${ }^{13}$ Some effort was recently devoted to exploiting the LC behavior to tune the film structure. ${ }^{14,15}$

Shear or liquid crystalline behavior directly affects the structure of wet films and membranes. However, solid films are used in actual devices and applications, requiring a drying step after wet deposition. Drying mechanisms are expected to play a significant role in the film structure beyond the initial possible ordering in the wet state. This is a critical issue especially for micrometer thick coatings, where drying induced inhomogeneity is likely to occur.

The drying of a colloidal dispersion is a quite complex process. Changes in concentration and viscosity and the setting up of instabilities are only some of the processes the film

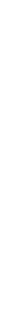


It is therefore critical to tune the formation of wrinkles and to develop a better understanding to control their structure or even to suppress them.

Nowadays, it is possible to induce controlled wrinkle formation through the application of known mechanical stresses to dried films. For instance, mono or few layer graphene films were transferred onto prestrained stretchable substrates. $^{25,26}$ As the strain was released, films started wrinkling. In another example, the strain appeared sponta neously when ultrathin graphite films were suspended on predefined trenches. ${ }^{27}$ In this latter case, a mechanical stress was also thermally induced by the mismatch of the thermal expansion coefficients of the graphene layer and that of the substrate. Concerning GO liquid crystalline systems, only few research efforts can be found in the literature. ${ }^{14,15}$ In the first case, ${ }^{14,19}$ the authors related the shear banding of the LC flow alignment to the wrinkles that appear after drying. In a more recent study on wrinkling, thick films were produced at high temperature $\left(>50{ }^{\circ} \mathrm{C}\right)$ and low blade coating velocities. ${ }^{15}$ Different types of wrinkles were observed as a function of the drying conditions.

Here, we show by using suspensions with different compositions that the rheological properties of the suspensions are key features in controlling the wrinkles and patterns formed upon drying.

We show in particular how the wavelength of the wrinkles varies with the composition of the GO solution, without any obvious link with pre existing ordering in the GO liquid crystal films and without using prestretched substrates. A correlation with mechanical and shear rheological properties of the GO dispersion is discussed. We demonstrate the possibility to pattern wrinkles through the development of an evaporative lithography method. ${ }^{28}$ The latter is shown to be negative, by contrast to other common colloid films, with the accumulation of particles in regions with lower evaporation rates. The distinctive negative evaporative lithography is also driven by the rheological properties of the GO dispersions. Finally, we show how the inclusion of spherical nanoparticles can be used to reduce, and even completely suppress, the formation of wrinkles, therefore providing a method to make perfectly flat GO films from drying solutions. When used alone, the spherical nanoparticles typically form films which crack upon drying. This mechanism which is opposite to buckling and wrinkling results from the tension of capillary bridges between the particles. Here this mechanism is used to balance the spontaneous tendency of GO films to wrinkle.

The present results therefore provide a comprehensive basis to better control and tune the formation and structure of wrinkles in GO based films.

\section{EXPERIMENTAL SECTION}

Materials. Commercial graphene oxide solutions in water from Graphenea were used. The graphene oxide concentration is $4.0 \mathrm{mg} /$ $\mathrm{mL}$. The flake lateral size is a few micrometers. Ludox HS 40 silica nanoparticles were used as additives. Dispersions are provided by Aldrich with a batch concentration of $40 \mathrm{wt} \%$ in water. The average nanoparticle diameter is $12 \mathrm{~nm}$.

Sample Preparation. GO concentrated dispersions were obtained with a two step centrifugation process. ${ }^{12}$ The first step was a mild centrifugation $(1400 \mathrm{~g}, 20 \mathrm{~min})$ used to remove possible aggregates. The collected dispersion was centrifuged for $45 \mathrm{~min}$ at $50000 \mathrm{~g}$. This second step allowed the separation of a concentrated LC pellet of graphene oxide in water. The concentration, determined through the dry extract, was $(4.3 \pm 0.3)$ wt $\%$. All the other concentrations were obtained by dilution in water. Care was taken to ensure homogeneous mixing after water addition. Usually, diluted samples were vortex mixed for at least $30 \mathrm{~s}$.

Nanoparticles and GO dispersions were obtained by mixing the two dispersions in a volume ratio of 1:10. After being mixed, the hybrid systems were bath sonicated for $15 \mathrm{~min}$.

Rheology. Rheological measurements were performed with an AR2000 stress controlled rheometer from TA Instruments. All of the samples were analyzed with $40 \mathrm{~mm}$ cone and plate geometry at 25.0 ${ }^{\circ} \mathrm{C}$. Evaporation was avoided by the use of a trap to keep the humidity rate constant during the measurements.

Film Preparation. GO films were prepared with a doctor blade combined with rod coating technology. A drop of dispersion was put on a glass substrate, and coating proceeded at a velocity, $v$, of $1 \mathrm{in.} / \mathrm{s}(2.5$ $\mathrm{cm} / \mathrm{s}$ ). Different velocities did not show any particular effect, so the slowest available was chosen. The average film size was $2.5 \times 1.5 \mathrm{~cm}^{2}$. To avoid geometrical issues, a constant amount of material was used to obtain films with the same surface and shape. ${ }^{29}$ Care was taken to avoid substrate inclination by fixing it with a tape and checking for planarity with a bubble level. The films were produced under controlled conditions of temperature and relative humidity of $26.0{ }^{\circ} \mathrm{C}$ and $35 \%$, respectively. The films were not removed until they were completely dry.

Dynamic Evaporative Lithography. Film drying was performed in a confined environment. Soon after coating, a mask was placed above the film, at a distance of $\sim 300 \mu \mathrm{m}$. The mask size was $2.0 \times 7.5$ $\mathrm{cm}^{2}$. The mask ensured evaporation in a well controlled manner. Then the mask was moved at a velocity of $5-10 \mu \mathrm{m} / \mathrm{s}$, according to the contact line recession speed. Faster or slower velocities caused only a small perturbation in wrinkle order, maintaining the wavelength and alignment the same.

Film Characterization. The films were characterized with optical and electronic microscopy. The thickness was determined through the use of an OM Contour GTK optical profilometer. Data were analyzed with the Vision 64 software under white light illumination vertical scanning interferometry mode (VSI). Films were cut at different points to assess the different regions and to get an average film thickness.

Wavelength Determination. The wrinkle spacing (i.e., wave length, $\lambda$ ) was determined through a Gaussian analysis of distributions of 100 measures of distances extracted from 5 pictures per sample. One dimensional fast Fourier transformation (FFT) of different linear profiles extracted from optical micrographs was also used to confirm the obtained results.

\section{RESULTS}

Film Casting. GO films were prepared from water based dispersions in the GO concentration range of $0.6-4.3 \mathrm{wt} \%$. Coating was performed in a controlled shear rate range, $\dot{\gamma}(=v /$ $\left.H_{\mathrm{w}}\right) \approx 100-500 \mathrm{~s}^{-1}$, where $v(2.5 \mathrm{~cm} / \mathrm{s})$ is the velocity of the moving blade and $H_{w}$ is the thickness of the wet film. The investigated systems showed shear thinning behavior over the whole range of tested shear rates in rheology characterizations. In confined geometries, the systems form shear bands, as already observed elsewhere. ${ }^{14}$ However, these flow induced structures rapidly disappeared during drying.

The progressive rearrangement of flakes and the setting up of concentration gradients produced wrinkled films with no controlled structures. Control over temperature and humidity conditions was not sufficient to avoid this problem. For this reason, we developed a technique to induce progressive directional evaporation. The choice was motivated by the need to control solvent flow to promote flake assembly and solid film growth along a particular direction. The technique is schematized in Figure 1. Soon after casting, the wet films were 

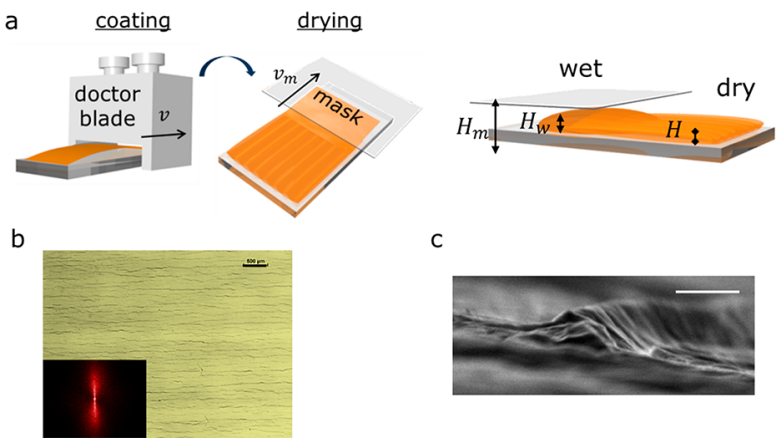

Figure 1. Film casting and drying is shown in (a) along with the overall wrinkle alignment (2D FFT inset) (b) and the microscopic appearance of a wrinkle (c). $v$ and $v_{\mathrm{m}}$ indicate the directions of blade and mask movement, and $H_{\mathrm{m}}, H_{\mathrm{w}}$, and $H$ are the mask, wet film, and dry film heights, respectively. Scale bars are (b) $500 \mu \mathrm{m}$ and (c) 500 nm.

covered with masks of different heights $H_{\mathrm{m}}$. The mask-wet film distance $\left(H_{\mathrm{m}}-H_{\mathrm{w}}\right)$ was fixed at $\sim 300 \mu \mathrm{m}$ to stop evaporation under the covered area. The end of the film was left uncovered to start evaporation. The films progressively dried as the mask was retracted at a constant velocity, $v_{\mathrm{m}}$. During the whole process, wrinkles (Figure 1c) on the dry film developed from the wavy surface of the wet part. The controlled drying allowed control over wrinkle patterns. An average alignment was obtained along the drying direction. The volume shrinkage of the film induced a compressive stress that caused elastic instabilities. As observed elsewhere, ${ }^{21}$ rehydration relaxed and suppressed the formed wrinkles. The wrinkles appeared birefringent under crossed polarizers.

Surface Wrinkling Characterization. As soon as the wet film started drying, wrinkles appeared on the surface at the free air-water interface (i.e., outside the mask). These surface wrinkles were compressed and folded after complete drying. This behavior is reminiscent of the behavior of polymeric systems ${ }^{30}$ for which a skin layer forms and buckles. The pinning of the contact line and surface tension on the (covered) wet side clamped the skin at these two ends (i.e., the wet and the dry film boundaries) of the receding front. Then, evaporation induced a reduction in volume associated with a compressive strain producing wrinkling. This process finally allowed the alignment of the wrinkles perpendicular to the receding liquid front. The spacing of parallel wrinkles was analyzed as indicated in the Experimental Section. In Figure 2, 1D FFTs taken from grayscale profiles of the microscopic pictures are shown. For all of the tested concentrations and film thicknesses, nearly the same spacing was found for wet and dry films from a given system, even though the spacing distribution appears broader for dry films.

Concentration and Thickness Effects. The wrinkle spacing, $\lambda$, was determined as a function of GO concentration and dry film thickness, $H$, as shown in Figure 3. First, the solid content of the dry film was kept constant. The wavelength was measured for films obtained from dispersions of different GO concentrations. To achieve this, the film area was kept constant, and $H_{\mathrm{w}}$ was changed proportionally to the GO concentration. As shown in Figure 3a, $\lambda$ decreases with a power law like decay at increasing dispersion concentration. Second, films of different $H$ (i.e., final solid content) were produced. A certain GO dispersion was deposited at different $H_{\mathrm{w}}$ values (i.e., different final deposit, $H$ ). The process was repeated for three different GO concentrations. In this second case, $\lambda$ was found to increase linearly with $H$ (Figure $3 \mathrm{~b}$ ). The slope of this linear increase changes with concentration.

Thin Films. Very thin films were prepared by a modification of the blade coating approach. A flexible scraper was attached to the blade to reproduce the technique used in ref 31 (inset of Figure 3c). At very low thickness (inset of Figure 3c), films showed colors from thin film interference, and no more wrinkles were detected. The thickness boundary $h$ between the wrinkling of thick films and no wrinkling of thin films was determined for different GO concentrations (Figure 3c). The absence of wrinkling in thin films reflects the absence of skin formation during drying. ${ }^{32,33}$ Actually because of its very thin structure, the film dries as a whole gel. Similar behavior was reported for polymer films. Following de Gennes, ${ }^{32}$ a skin forms when the polymer concentration in the top layer of an evaporating solution increases above a given value. A steady state solvent evaporative current is then established in the skin.
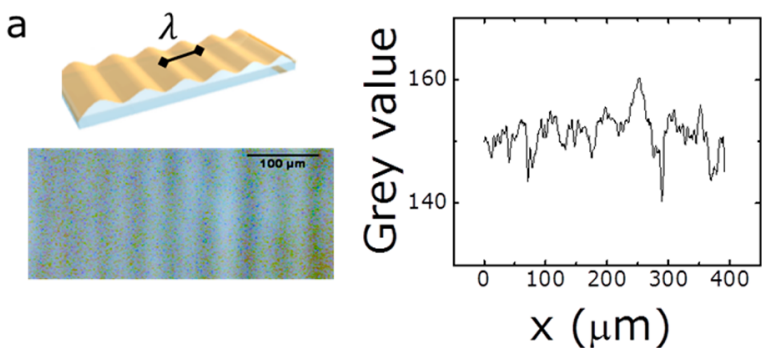

b

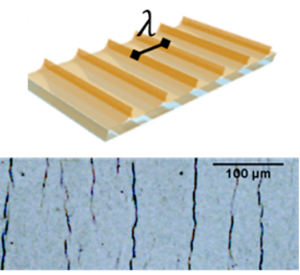

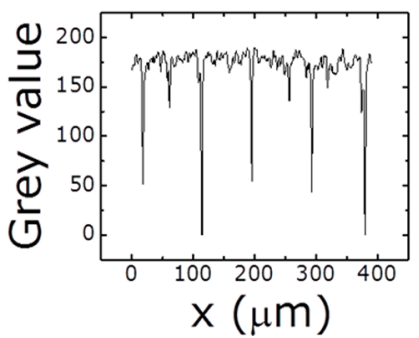
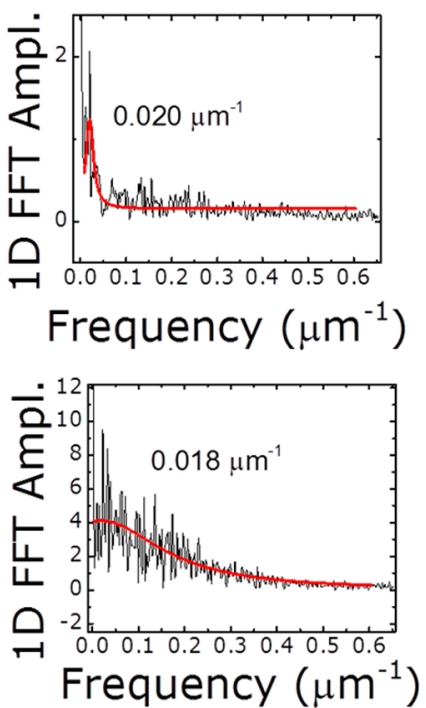

Figure 2. Wavelength determination examples, optical micrograph, grayscale profile, and 1D FFT for the wet (a) and dry (b) films. The scale bar is $100 \mu \mathrm{m}$. 
a

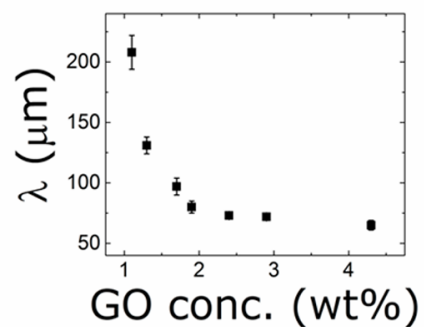

b

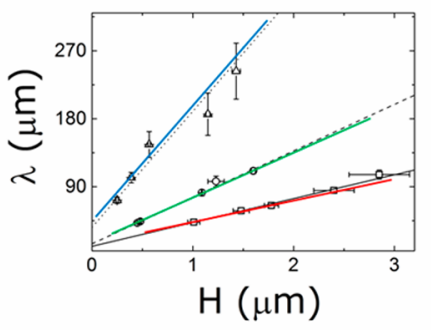

C

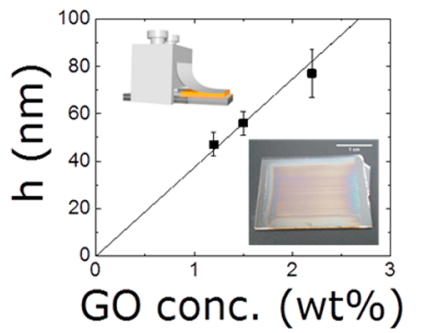

Figure 3. $\lambda(\mu \mathrm{m})$ is reported as a function of GO wt \% at a fixed $(1.3 \pm 0.5 \mu \mathrm{m})$ dry thickness (a) and (b) at different dry thicknesses $(H, \mu \mathrm{m})$ for $4.3 \mathrm{wt} \%$ (square, red line), $1.7 \mathrm{wt} \%$ (circle, green line), $0.68 \mathrm{wt} \%$ (triangle, blue line) GO dispersions. (c) Thin film thickness, $h$ (nm), as a function of GO wt \%. (Insets) The blade with a flexible scraper and the appearance of a film (scale bar $1.0 \mathrm{~cm})$.

By comparing solvent diffusion in the vapor phase with that through the skin, he determined a limiting skin thickness $\left(\tau_{0}\right)$ of around $70 \mathrm{~nm}$. Therefore, for films of comparable thickness a steady state solvent evaporation is set throughout the whole film height, and no skin formation is expected. The measured $h$ was actually in the same range for polymer systems, representing a good approximation of the skin layer $(\tau)$ of a (hypothetical) thick film (i.e., $h \approx \tau$ ).

Nanoparticle Effect. The effect of added spherical nanoparticles on film structure was investigated. First, it was checked that the nanoparticles had no effect on the GO dispersion phase behavior in the concentration range of the present study. No phase separation or destabilization was observed for weeks. Then, films were prepared at a fixed $H_{\mathrm{w}}$ of $40 \mu \mathrm{m}$. A map of the different film structures is reported in Figure 4. Three different situations were observed: wrinkling
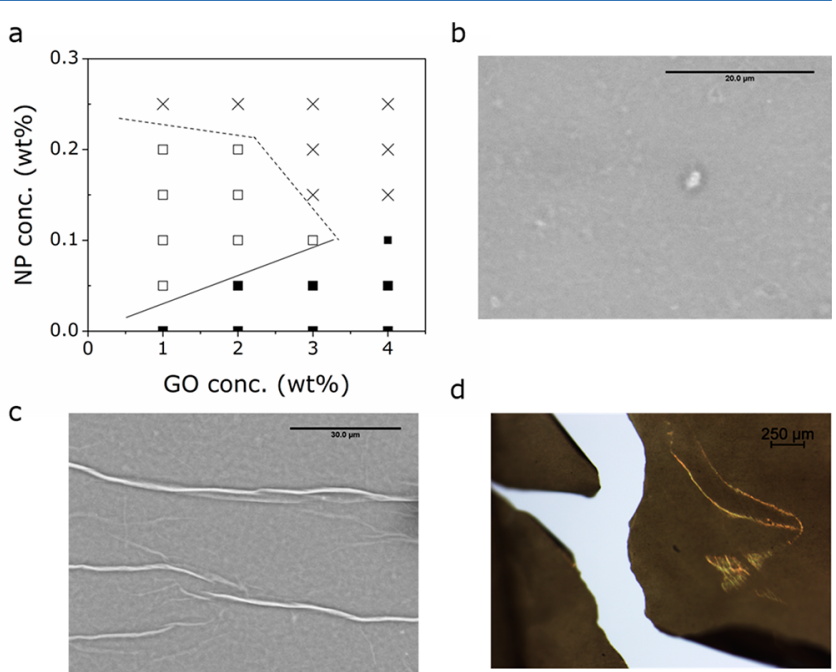

Figure 4. Effect of NP addition. (a) Wrinkling phase diagram for hybrid films at $40 \mu \mathrm{m}$ fixed $H_{\mathrm{w}}$. Wrinkled (black squares), smooth (empty square), and cracked (cross) films. (b, c) SEM and (d) optical images of smooth, wrinkled, and cracked films, respectively. Scale bars are $20(\mathrm{~b}), 30$ (c), and $250 \mu \mathrm{m}(\mathrm{d})$.

(filled squares), flattening (open squares), and cracking (crosses). Under certain conditions of GO and NP concentrations, wrinkling was completely suppressed. The addition of NPs favored the casting of smoother films. The threshold concentration of NPs to remove wrinkling increased with GO concentration. Above a certain threshold of NP content, films underwent cracking. At the highest GO concentration used, it was not possible to reduce wrinkling without cracking the dry film.

Controlled Patterns. By using evaporative lithography, it is possible to control not only wrinkle alignment but also the formation of particular patterns. Two examples are reported in Figure 5. Films were dried under a fixed holed mask. The 1

a

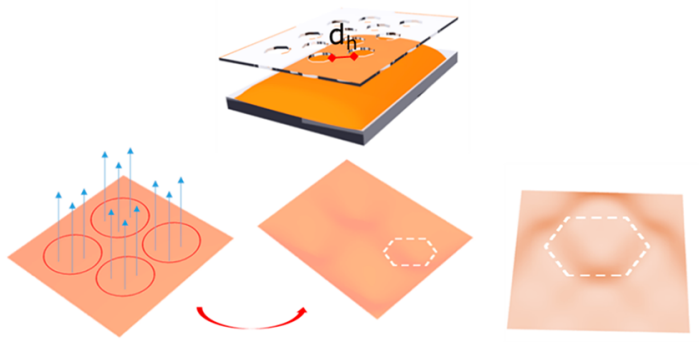

b

C
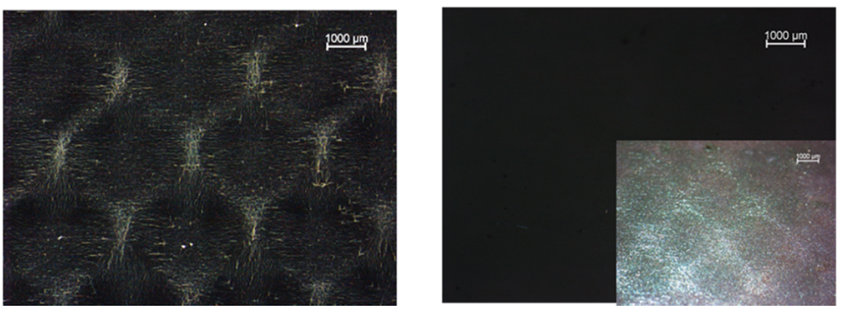

Figure 5. Patterning of GO (2.0 wt \%) and GO + NPs (2.0 and $0.1 \mathrm{wt}$ $\%$, respectively) films: (a) schematic representation of the holed mask with the drying and patterning scheme; cross polarizing micrographs showing the obtained patterns for (b) pure GO and (c) GO NPs film. (Inset) Optical micrograph for the hybrid films, obtained in reflection. The scale bar is $1000 \mu \mathrm{m}$.

$\mathrm{mm}$ diameter holes were hexagonally arranged with an average pitch of $d_{\mathrm{h}} \leq 1 \mathrm{~mm}$. The mask pattern was accurately reproduced on the films. Circular menisci were first formed under the open holes, where evaporation was higher. The meniscus diameter grew with time. Finally, different menisci joined under the covered part of the mask. This differential evaporation was responsible for the formation of hexagonal features with higher edges. Surprisingly, this behavior differs from conventional colloidal evaporative lithography. The present case is peculiar to a negative evaporative lithography, as the higher features were cast under the covered part, in contrast to the case of conventional nanoparticle aqueous dispersions. $^{28}$ The reverse situation was reported only in solvent mixtures (i.e., water-ethanol), when Marangoni effects play a role. ${ }^{34}$ 
The same peculiar patterns were reproduced in pure GO as well as in GO NP hybrid systems. However, the presence of NPs completely removed wrinkles. This was particularly evident through cross polarizing microscopy. Hybrid films did not show any birefringence because of the absence of wrinkles. The wrinkle arrangement was the consequence of the stress field arising from uneven evaporation rates. Inside the open holes, small wrinkles were aligned perpendicularly with respect to the meniscus contact line, while higher crumpled regions appeared under the covered parts. In the hybrid systems, no small wrinkles were detected, while higher crumpled deposits were still present. The overall pattern appeared more blurred.

\section{DISCUSSION}

Wrinkling is a universal phenomenon when applying a compression/tension to elastic films. The general theory of Cerda and Mahadevan ${ }^{35}$ defines the wrinkle wavelength, $\lambda$, as follows

$$
\lambda \approx\left(\frac{B}{K}\right)^{1 / 4}
$$

where $B$ is the film bending stiffness $\left(=E_{\mathrm{f}} \tau^{3}, E_{\mathrm{f}}\right.$ is Young's modulus, and $\tau$ is the film thickness) and $K$ is the stiffness of an effective elastic foundation. It is possible to address a particular case by knowing the physics of the system. The present system is composed of a rigid film lying on an elastic support made of the GO suspension in a gel like liquid crystal state. In that case, $K$ takes the form of an elastic bulk modulus, giving

$$
\lambda \approx \tau\left(\frac{E_{\mathrm{f}}}{E_{\mathrm{s}}}\right)^{1 / 3}
$$

The wavelength, $\lambda$, is proportional to the top layer thickness, $\tau$, with a slope defined by the mismatch of the film, $E_{f}$, and substrate, $E_{s}$, Young's moduli. GO exhibits surfactant like behavior, exposing the hydrophobic moiety at the air-water interface. ${ }^{36}$ In particular, the used GO possess the proper $\mathrm{C} / \mathrm{O}$ ratio to be easily entrapped in the interface. ${ }^{37}$ In the used concentration range, due to their large lateral size, flake diffusion is limited. If the evaporation is quite fast, GO flakes tend to accumulate at the interface. Routh and Zimmerman ${ }^{38}$ defined a specific Peclet number, $P e \approx H_{w} \dot{E} / D$, to quantify the ratio between diffusion $(D)$ and the evaporation rate $(\dot{E})$. At $P e$ $\gg 1$, evaporation overtakes diffusion and skinning is expected. Approximating $\dot{\mathrm{E}}$ as the water front velocity $(\sim \mu \mathrm{m} / \mathrm{s})$ and the diffusion coefficient as that of a sphere of $1 \mu \mathrm{m}$ size, one obtains $P e \approx 8$, fulfilling the above conditions. Thus, a skin layer is expected to form on the top of the film. An analogous process was already observed in polymeric systems. ${ }^{39}$ The high frictional forces among the flakes and the pinning of the contact line suppress slippage, and the skin is finally folded under compression. ${ }^{21}$ The resultant in plane compressive stress is directed by the receding water front. In this hypothesis, eq 2 could explain the different dependencies shown in Figure 3. A skin layer of thickness $\tau$ is suspended on foundations with different elasticities (i.e., GO bulk dispersions). The shear elastic modulus $\left(G^{\prime}\right)$ of bulk GO dispersions increases with concentration, as observed by rheology experiments. In principle, knowledge of the Poisson ratio for the GO foundation layer allows the determination of the bulk Young modulus, $E_{s}$. However, the qualitative trend in $E_{\mathrm{s}}$ with concentration can still be defined by a rough approximation, $\mathrm{Es} \approx \mathrm{G}^{\prime}$.
Using eq 2, an apparent bending stiffness $B$ and its dependence on concentration can be inferred from the data in Figure 3, as shown in Figure 6.

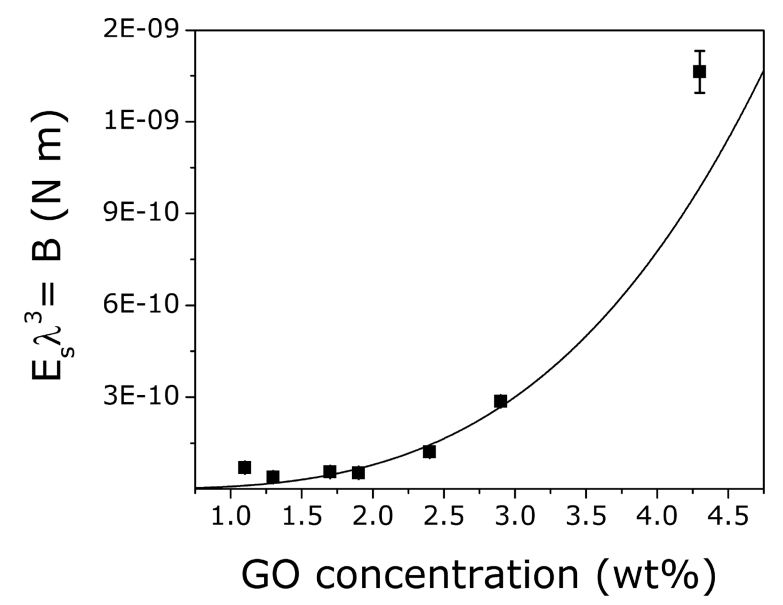

Figure 6. Bending stiffness $\left(B=E_{\mathrm{s}} \lambda^{3}, \mathrm{~N} \mathrm{~m}\right)$ reported as a function of GO wt \%.

This bending stiffness increases as a power law $\left(B \approx \operatorname{conc}^{\mathrm{b}}\right)$ with an exponent $b$ close to 3 (3.3). Considering the relation $B$ $=E_{\mathrm{f}} \tau^{3}$, the behavior can be explained by assuming a direct proportionality of skin thickness, $\tau$, with GO concentration. If one considers a Young modulus on the order of $\sim 10^{2} \mathrm{GPa},{ }^{40}$ then a skin layer in the range of $10-100 \mathrm{~nm}$ is expected. These considerations are actually in agreement with data obtained for very thin films (Figure 3c). The approximations of the Young and bending moduli are quite different from that obtained for monolayer graphene oxide. ${ }^{37}$ However, the present situation concerns multilayer assembly and the whole mechanical properties derived in a complex way from the monolayer. Compressive rheology characterization would be more appropriate in this particular situation. ${ }^{41}$ However, shear and compression properties have been shown to behave in qualitatively the same manner. ${ }^{42}$ Therefore, the considerations above can give an idea of our wrinkling mechanics. Unfortunately, characterization of the skin layer is challenging, and a complete quantitative characterization of the film is not possible.

Wrinkle alignment is a consequence of a resultant tensile stress applied perpendicularly to the drying front as the volume decreased with the contact lines of the skin pinned. The mask drives the formation of a horizontal drying front. This front separates the dry film from the liquid film under the mask. A skin on the drying front is pinned at the contact line and stretched backward by the liquid surface tension. These constraints drive a unidirectional tensile stress in response to the normal compression due to volume shrinkage. The shape of the mask assures negligible skin formation at the lateral sides controlling the alignment.

The addition of nanoparticles modifies the physics of the system. In particular, we are interested here in particles that form cracks when used alone, a behavior exactly opposite to the case of GO systems which have extra surface area. The relatively low nanoparticles concentration presently used does not affect the phase behavior and the bulk mechanical properties of the dispersions. But other effects are coming into play. As known, above a critical thickness, films made solely of nanoparticles tend to crack. 
During evaporation, a close packed configuration of NPs forms at the contact line. As the solvent recedes (vertically) from this NP front, a large negative capillary pressure is produced in the menisci between particles. ${ }^{43}$ This generates a compression stress normal to the surface and a large in plane tensile stress, as the rigid substrate prevents lateral deformation. Crack formation will release the tensile stress, at the expense of surface energy. The present situation is more complicated due to the presence of two different particles with two limiting distinct behaviors, one with the formation of cracks and the other with the formation of wrinkles. Actually, it was observed that the in plane stress induced by NPs at the surface of the films could even balance the stress that normally generates wrinkles. This can result in the total disappearance of wrinkles. The large size difference between flakes and nanoparticles is expected to produce stratification. With the same Pe argument for skin formation, one can expect an uneven distribution of flakes and nanoparticles during drying. ${ }^{16}$ In a recent simulation, ${ }^{44}$ the distribution of NPs in a skinning polymer solution was attributed mostly to NP-polymer and NPsolvent interactions. The nanoparticles used here are hydro philic and have a better affinity for the solvent. In this case, NPs are expected to accumulate under the superficial skin. Actually, no NPs are visible at the top of the dry films in experiments. During evaporation, volume shrinkage will set NP layers under compression. As the NPs are not deformable and their movement is hindered under confinement (i.e., GO stack assembly), in plane tensile stress is produced. This stress will first flatten the GO skin layer until there is enough disposable surface from the wrinkles, then again producing cracks. We can infer that the shape of the boundary lines comes from a complex interplay of wrinkle amplitude, wavelength, and dry thickness effects.

The possibility of patterning a peculiar structure with negative lithography can be explained by considering the yield stress nature of GO liquid crystal dispersions. ${ }^{12}$ Menisci are created under the open areas of the mask due to solvent loss (Figure 5a). The stress generated by a Laplace pressure gradient $(\sigma=2 \gamma / R \approx 14 \mathrm{~Pa}$, for water surface tension and hole size $R=1 \mathrm{~mm}$ ) is not high enough to overtake the yield stress (i.e., $\sigma_{y}>14 \mathrm{~Pa}$ for $2.0 \mathrm{wt} \% \mathrm{GO}, \mathrm{SI}$ ) and to induce viscous flow. Therefore, the films will retain the resulting deformation, generating the pattern. As already mentioned, a compressive yield stress would be more appropriate, but the consideration still holds.

\section{CONCLUSIONS}

By taking advantage of dynamic evaporative lithography and tuning the rheological properties of GO dispersions, we were able to control the wrinkling of GO films. The obtained wrinkles were aligned over a macroscopic area of different GO deposits. The wavelength was tuned by changes in the concentration and thickness of the films. The phenomenon was attributed to the formation of a skin layer, subjected to compressive strain during drying. It was shown that this compressive stress was balanced with a tensile stress to get rid of the wrinkles. The latter was simply obtained with the addition of nanoparticles, making the concept easily imple mentable in applications.

Controlling the phenomenon of wrinkling is critical in the fabrication of particular patterns, as illustrated in the present work. Notably, wrinkling can be altered without affecting the final macroscopic texture. This is actually the case of evaporative lithography in hybrid systems. This is important since wrinkles can affect the properties of the films.

As already mentioned, ${ }^{24}$ the relation between GO film roughness and its antimicrobial activity has been demonstrated. We expect that tuning the wrinkle spacing may add selectivity. Patterned films can be also used as templates for controlled nanoparticle deposition. In principle, anisotropic wettability may also be obtained.

There are still some open questions related to the physical mechanisms involved in the investigated phenomena. We used a modification of the elasticity theory to qualitatively describe the mechanics of the system. The foundation considered in the theory is a purely elastic material, whereas in our case the material is viscoelastic. Moreover, the whole process is dynamic, as the top layer is forming and growing continuously. Therefore, more accurate theories are needed to consider all of these phenomena. From an experimental point of view, the characterization of the skin layer would also be interesting. The determination of the skin thickness will allow the confirmation of our hypothesis. It would also be interesting to look at the NPs' spatial distribution during film drying. The character ization of NPs and their packing would be helpful in quantifying the stress built up during drying.

\section{AUTHOR INFORMATION}

\section{Corresponding Author}

*E mail: franco.tardani@gmail.com.

ORCID

Franco Tardani: 0000000292981947

\section{Present Addresses}

${ }^{\dagger}$ LOMA, Laboratoire Ondes et Matiere d'Aquitaine (UMR 5798), Universite de Bordeaux-CNRS, 33405 Talence, France.

${ }^{\ddagger}$ ESPCI, CNRS, SIMM UMR 7615, 11 rue Vauquelin, 75005 Paris, France.

\section{Author Contributions}

F.T., supervised by W.N., performed the experimental part of this article. The flexible scraper experiments were performed under the supervision of H.K. P.P directed the project in collaboration with A.C. and C.Z. The article was written through the contributions of all authors.

\section{Funding}

The research in the manuscript was conducted in the framework of the A.N.R. funded GAELIC project.

\section{Notes}

The authors declare no competing financial interest.

\section{ACKNOWLEDGMENTS}

We acknowledge C. Blanc, collaborator at the GAELIC project, for fruitful discussions and E. Laurichesse for some technical support of the experimental part. 


\section{REFERENCES}

(1) Eda, G.; Chhowalla, M. Chemically Derived Graphene Oxide: Towards Large Area Thin Film Electronics and Optoelectronics. Adv. Mater. 2010, 22, 2392-2415.

(2) Yan, C.; Cho, J. H.; Ahn, J. H. Graphene based flexible and stretchable thin film transistors. Nanoscale 2012, 4, 4870-4882.

(3) Chee, W. K.; Lim, H. N.; Zainal, Z.; Huang, N. M.; Andou, Y. Flexible Graphene Based Supercapacitors: A Review. J. Phys. Chem. C 2016, 120, 4153-4172.

(4) Chang, H.; Wu, H. Graphene Based Nanomaterials: Synthesis, Properties, and Optical and Optoelectronic Applications. Adv. Funct. Mater. 2013, 23, 1984-1997.

(5) Liu, G.; Jin, W.; Xu, N. Graphene based membranes. Chem. Soc. Rev. 2015, 44, 5016-5030.

(6) Kumar, S.; Chatterjee, K. Comprehensive Review on the Use of Graphene Based Substrates for Regenerative Medicine and Biomedical Devices. ACS Appl. Mater. Interfaces 2016, 8, 26431-26457.

(7) Chua, C. K.; Pumera, M. Chemical reduction of graphene oxide: a synthetic chemistry viewpoint. Chem. Soc. Rev. 2014, 43, 291-312.

(8) Pei, S.; Cheng, H. M. The reduction of graphene oxide. Carbon 2012, 50, 3210-3228.

(9) Aboutalebi, S. H.; Goudarzi, M. M.; Zheng, Q. B.; Kim, J. K. Spontaneous Formation of Liquid Crystals in Ultralarge Graphene Oxide Dispersions. Adv. Funct. Mater. 2011, 21, 2978-2988.

(10) Xu, Z.; Gao, C. Aqueous Liquid Crystals of Graphene Oxide. ACS Nano 2011, 5, 2908-2915.

(11) Kim, J. E.; Han, T. H.; Lee, S. H.; Kim, J. Y.; Ahn, C. W.; Yun, J. M.; Kim, S. O. Graphene Oxide Liquid Crystals. Angew. Chem., Int. Ed. 2011, 50, 3043-3047.

(12) Poulin, P.; Jalili, R.; Neri, W.; Nallet, F.; Divoux, T.; Colin, A.; Aboutalebi, S. H.; Wallace, G.; Zakri, C. Superflexibility of graphene oxide. Proc. Natl. Acad. Sci. U. S. A. 2016, 113, 11088-11093.

(13) Diat, O.; Roux, D.; Nallet, F. Effect of shear on a lyotropic lamellar phase. J. Phys. II 1993, 3, 1427-1452.

(14) Luo, Y.; Bragging, G. A.; Olson, G. T.; Stevenson, A. R.; Ruan, W. L.; Zhang, S. Nematic Order Drives Macroscopic Patterns of Graphene Oxide in Drying Drops. Langmuir 2014, 30, 14631-14637.

(15) Hong, S. H.; Shen, T. Z.; Song, J. K. Controlling wrinkles and assembly patterns in dried graphene oxide films using lyotropic graphene oxide liquid crystals. Liq. Cryst. 2017, 44, 939-947.

(16) Routh, A. Drying of thin colloidal films. Rep. Prog. Phys. 2013, 76, 046603 .

(17) Deegan, R. D.; Bakajin, O.; Dupont, T. F.; Huber, G.; Nagel, S. R. Witten, T. A. Capillary flow as the cause of ring stains from dried liquid drops. Nature 1997, 389, 827-829.

(18) Singh, K. B.; Tirumkudulu, M. S. Cracking in Drying Colloidal Films. Phys. Rev. Lett. 2007, 98, 218302.

(19) Hong, S. H.; Shen, T. Z.; Song, J. K. Water front recession and the formation of various types of wrinkles in dried graphene oxide droplets. Carbon 2016, 105, 297-304.

(20) Ahmad, R. T. M.; Hong, S. H.; Shen, T. Z.; Song, J. K. Water assisted stable dispersal of graphene oxide in non dispersible solvents and skin formation on the GO dispersion. Carbon 2016, 98, 188-194.

(21) Guo, F.; Kim, F.; Han, T. H.; Shenoy, V. B.; Huang, J.; Hurt, R. $\mathrm{H}$. Hydration Responsive Folding and Unfolding in Graphene Oxide Liquid Crystal Phases. ACS Nano 2011, 5, 8019-8025.

(22) Cote, L. J.; Kim, J.; Zhang, Z.; Sun, C.; Huang, J. Tunable assembly of graphene oxide surfactant sheets: wrinkles, overlaps and impacts on thin film properties. Soft Matter 2010, 6, 6096-6101.

(23) Wang, Z.; Tonderys, D.; Leggett, S. E.; Williams, E. K.; Kiani, M. T.; Steinberg, R. S.; Qiu, Y.; Wong, I. Y.; Hurt, R. H. Wrinkled, wavelength tunable graphene based surface topographies for directing cell alignment and morphology. Carbon 2016, 97, 14-24.

(24) Zou, F.; Zhou, H.; Jeong, D. Y.; Kwon, J.; Eom, S. U.; Park, T. J.; Hong, S. W.; Lee, J. Wrinkled Surface Mediated Antibacterial Activity of Graphene Oxide Nanosheets. ACS Appl. Mater. Interfaces 2017, 9, 1343-1351.
(25) Zang, J.; Ryu, S.; Pugno, N.; Wang, Q.; Tu, Q.; Buehler, M. J.; Zhao, X. Multifunctionality and control of the crumpling and unfolding of large area graphene. Nat. Mater. 2013, 12, 321-325.

(26) Thomas, A. V.; Andow, B. C.; Suresh, S.; Eksik, O.; Yin, J.; Dyson, A. H.; Koratkar, N. Controlled Crumpling of Graphene Oxide Films for Tunable Optical Transmittance. Adv. Mater. 2015, 27, 32563265.

(27) Bao, W.; Mao, F.; Chen, Z.; Zhang, H.; Jang, W.; Dames, C.; Lau, C. N. Controlled ripple texturing of suspended graphene and ultrathin graphite membranes. Nat. Nanotechnol. 2009, 4, 562-566.

(28) Harris, D. J.; Hu, H.; Conrad, J. C.; Lewis, J. A. Patterning Colloidal Films via Evaporative Lithography. Phys. Rev. Lett. 2007, 98, 148301.

(29) Kassuga, T. D.; Rothstein, J. P. The effect of shear and confinement on the buckling of particle laden interfaces. J. Phys.: Condens. Matter 2016, 28, 025101-11.

(30) Pauchard, L.; Allain, C. Mechanical instability induced by complex liquid desiccation. C. R. Phys. 2003, 4, 231-239.

(31) Deblais, A.; Harich, R.; Colin, A.; Kellay, H. Taming contact line instability for pattern formation. Nat. Commun. 2016, 7, 12458.

(32) De Gennes, P. G. Solvent evaporation of spin cast films: "crust" effects. Eur. Phys. J. E: Soft Matter Biol. Phys. 2002, 7, 31-34.

(33) Bornside, D. E.; Macosko, C. W.; Scriven, L. E. Spin coating: One dimensional model. J. Appl. Phys. 1989, 66, 5185-5193.

(34) Harris, D. J.; Lewis, J. A. Marangoni Effects on Evaporative Lithographic Patterning of Colloidal Films. Langmuir 2008, 24, 3681368.

(35) Cerda, E.; Mahadevan, L. Geometry and Physics of Wrinkling. Phys. Rev. Lett. 2003, 90, 074302.

(36) Kim, J.; Cote, L. J.; Kim, F.; Yuan, W.; Shull, K. R.; Huang, J. Graphene Oxide Sheets at Interfaces. J. Am. Chem. Soc. 2010, 132, $8180-8186$.

(37) Imperiali, L.; Liao, K. H.; Clasen, C.; Fransaer, J.; Macosko, C. W. Interfacial Rheology and Structure of Tiled Graphene Oxide Sheets. Langmuir 2012, 28, 7990-8000.

(38) Routh, A. F.; Zimmerman, W. B. Distribution of particles during solvent evaporation from films. Chem. Eng. Sci. 2004, 59, 2961-2968.

(39) Ciampi, E.; McDonald, P. J. Skin Formation and Water Distribution in Semicrystalline Polymer Layers Cast from Solution: A Magnetic Resonance Imaging Study. Macromolecules 2003, 36, 83988405.

(40) Jimenez Rioboo, R. J.; Climent Pascual, E.; Diez Betriu, X.; Jimenez Villacorta, F.; Prieto, C.; De Andres, A. Elastic constants of graphene oxide few layer films: correlations with interlayer stacking and bonding. J. Mater. Chem. C 2015, 3, 4868-4875.

(41) de Kretster, R. G.; Boger, D. V.; Scales, P. J. Compressive rheology: an overview. Rheol. Rev. 2003, 125-165.

(42) Zhou, Z.; Scales, P. J.; Boger, D. V. Chemical and physical control of the rheology of concentrated metal oxide suspensions. Chem. Eng. Sci. 2001, 56, 2901-2920.

(43) Lee, W. P.; Routh, A. F. Why Do Drying Films Crack? Langmuir 2004, 20, 9885-9888.

(44) Cheng, S.; Grest, G. S. Dispersing Nanoparticles in a Polymer Film via Solvent Evaporation. ACS Macro Lett. 2016, 5, 694-698. 